\title{
Paper-Based Triboelectric Nanogenerators Made of Stretchable Interlocking Kirigami Patterns
}

\author{
Changsheng Wu, ${ }^{\dagger}$, Xin Wang $^{\dagger,}$ Long Lin, ${ }^{\dagger}$ Hengyu Guo,${ }^{\dagger}$ and Zhong Lin Wang ${ }^{* \dagger,}$ \\ ${ }^{\dagger}$ School of Materials Science and Engineering, Georgia Institute of Technology, Atlanta, \\ Georgia 30332-0245, USA \\ ${ }^{\perp}$ Beijing Institute of Nanoenergy and Nanosystems, Chinese Academy of Sciences; National \\ Center for Nanoscience and Technology (NCNST), Beijing, 100083, P. R. China \\ *Address correspondence to zlwang@gatech.edu \\ *These authors contributed equally.
}


(a)

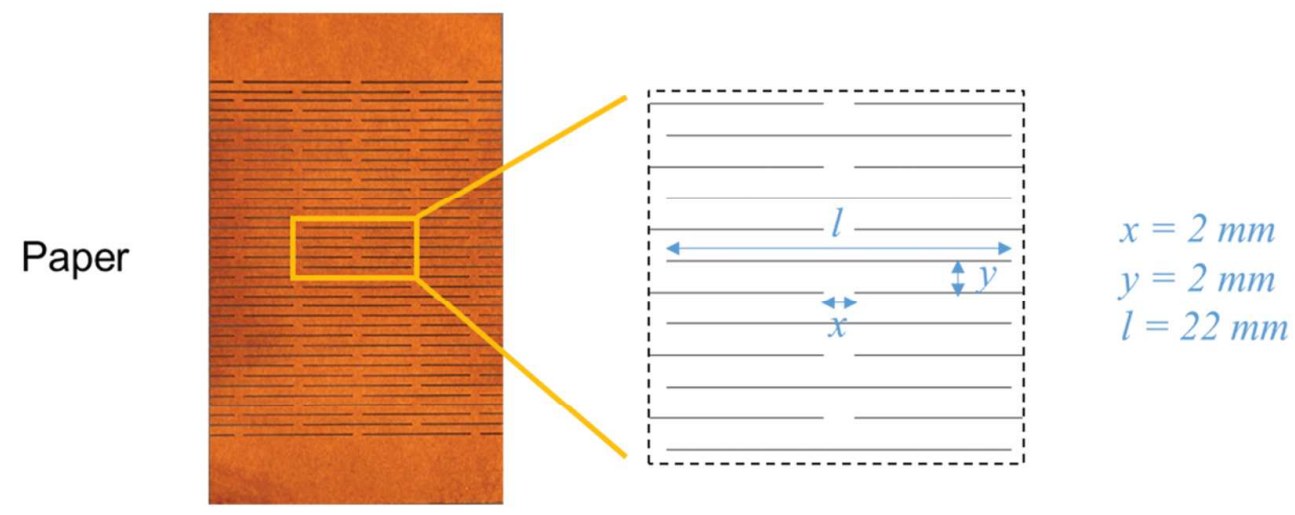

(b)

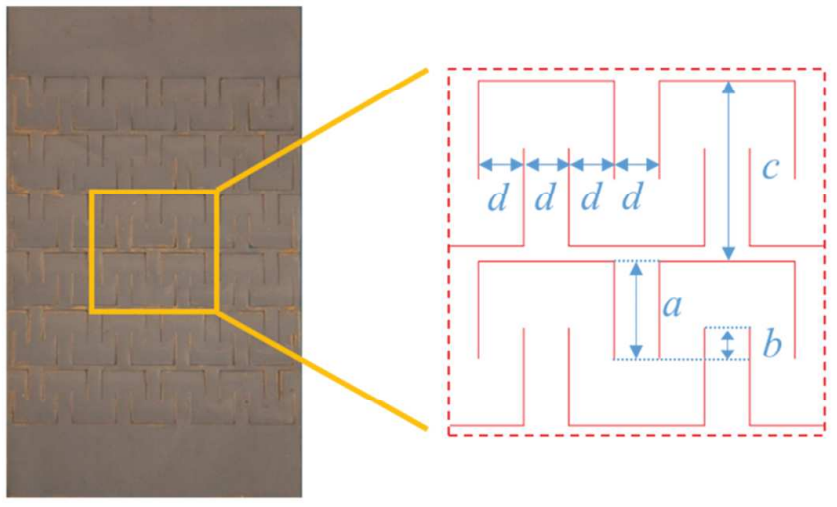

$$
\begin{aligned}
& a=6.5 \mathrm{~mm} \\
& b=2 \mathrm{~mm} \\
& c=12 \mathrm{~mm} \\
& d=3 \mathrm{~mm}
\end{aligned}
$$

c)

\section{Assembled Device}
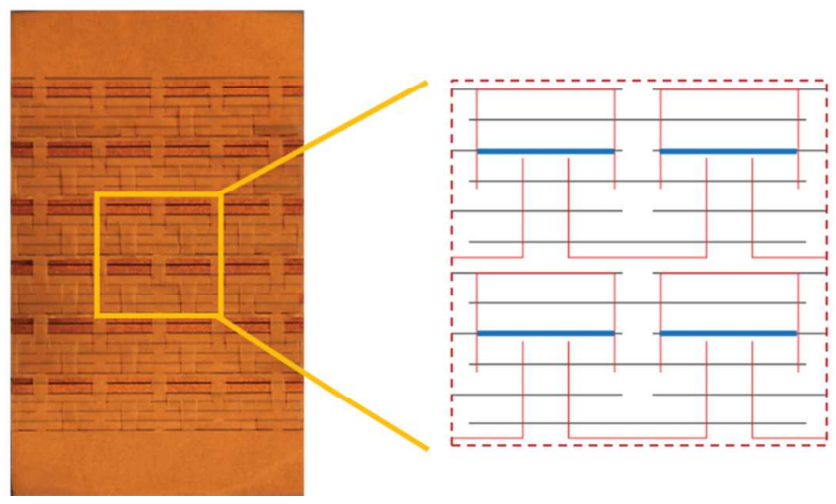

Inserted to paper back through blue lines

Figure S1. (a) Designed linear kirigami pattern for the Cu-paper. (b) Designed rectangular kirigami pattern for the thin films. (c) Assembly schematic of the interlocking structure. 


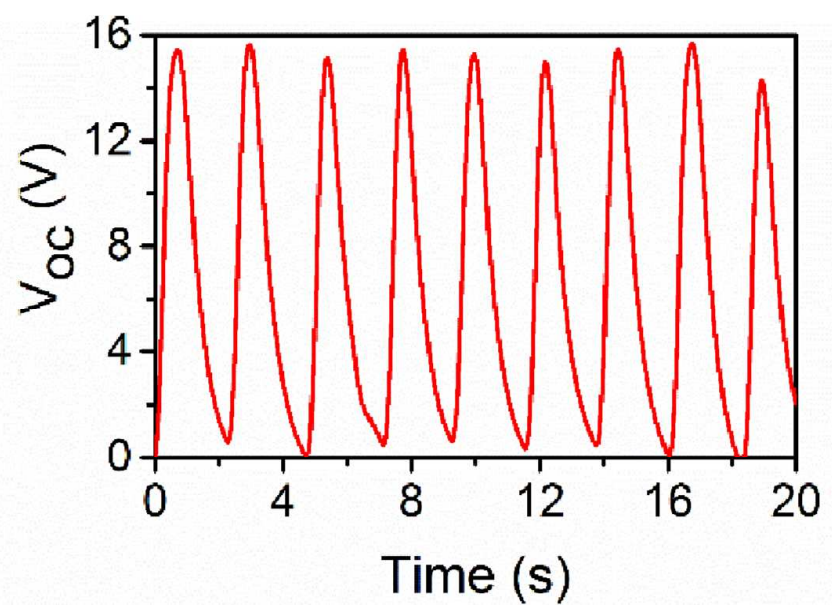

Figure S2. The open-circuit voltage of the FEP K-TENG device under indirect pressing mode. The device was covered by a piece of lightweight paper and another object covered with paper was used to apply the pressing force. The comparison with the output of direct pressing in Figure 5(e) suggests that the direct contact and separation between the device and the hand greatly enhances the output under the pressing mode; while the comparison with the output of stretching in Figure 5(b) suggests that the more intimate contact between the FEP and the Cupaper under pressing is another reason for the enhance output of the pressing mode compared to those of the stretching and twisting modes. 
(a)
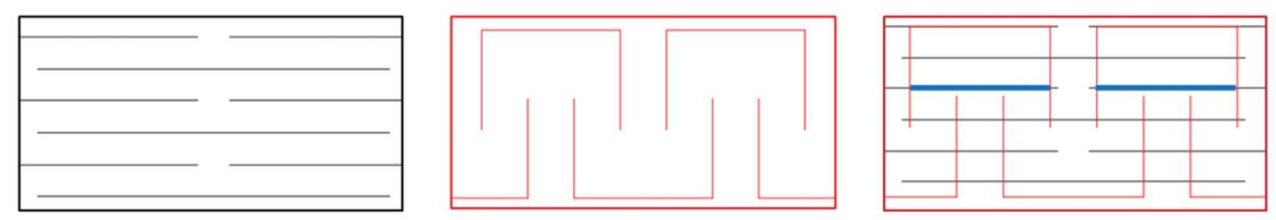

(b)

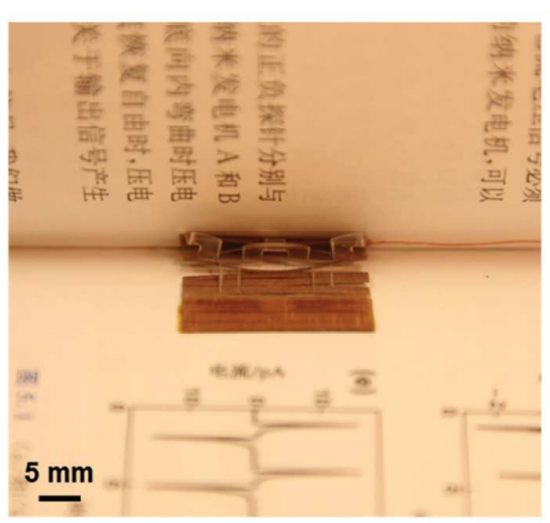

(c)

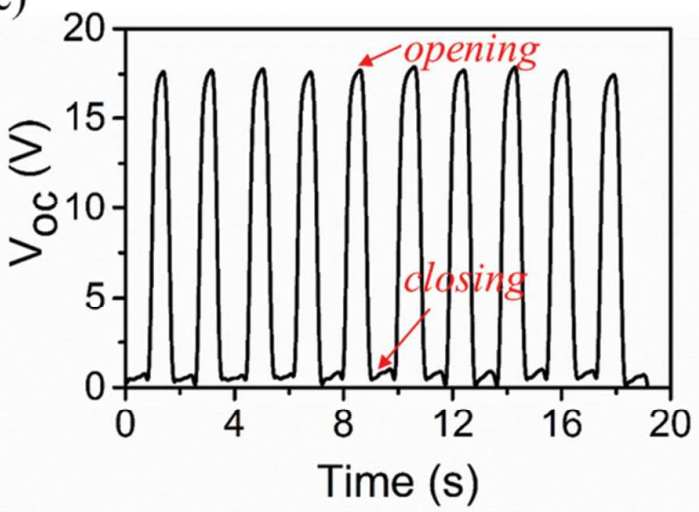

Figure S3. (a) Design of the self-powered sensor for the detection of book opening and closing. (b) Photograph of the sensor when attached to a book. (c) Typical sensing signal from book opening and closing. 


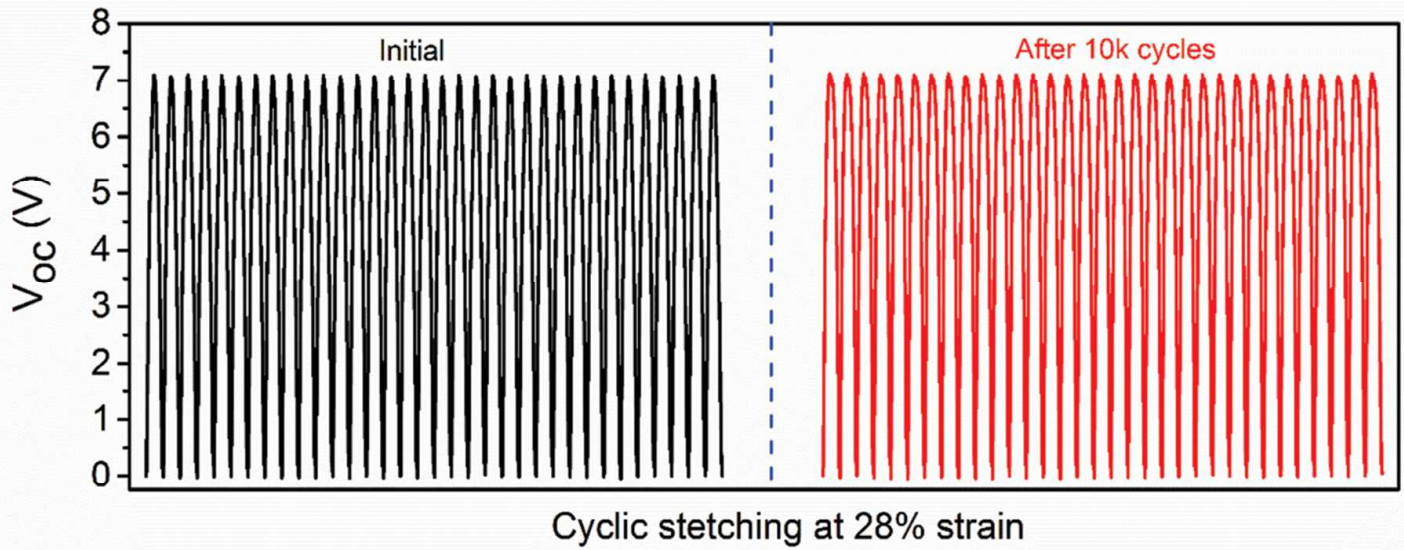

Figure S4. The open-circuit voltage of the FEP K-TENG device under cyclic stretching at $28 \%$ strain before and after 10,000 cycles. 
Movie 1. The application of the paper-based K-TENG for powering a small LCD screen.

Movie 2. The application of the paper-based K-TENG for powering LED lights.

Movie 3. The application of the paper-based K-TENG for detection of book opening and closing. 